\title{
Cerebrocostomandibular syndrome in four sibs, two pairs of twins
}

\author{
Vassiliki Drossou-Agakidou, Alexandros Andreou, Vassiliki Soubassi-Griva, \\ Maria Pandouraki
}

\begin{abstract}
We report four sibs, two pairs of twins, with cerebrocostomandibular syndrome (CCMS). The family history was negative. All four babies had the characteristic features of CCMS, including Pierre-Robin anomalad and rib dysplasia. Cerebral involvement was evident in two of the patients who had suffered perinatal asphyxia. The presence of the syndrome in all four sibs together with the negative family history in previous generations is consistent with Mendelian autosomal recessive inheritance with high penetrance.
\end{abstract}

The cerebrocostomandibular syndrome (CCMS) is a rare dysmorphic syndrome first described by Smith et $a^{1}$ in 1966. At least 34 cases of the syndrome have been reported to date. ${ }^{23}$ It is characterised primarily by defective costal development and features of the Pierre-Robin anomalad. ${ }^{12}$ Cerebral maldevelopment or malfunction is common among the reported cases. ${ }^{2}$ We describe the syndrome in four sibs, consisting of two pairs of twins. To our knowledge, this is the first report of CCMS in twins.

\section{Case reports}

The mother was a 23 year old primigravida at the birth of the first pair of twins. The father was 28

Department of Neonatology and Neonatal Intensive Care Unit, Aristotelian University of Thessaloniki, Greece.

V Drossou-Agakidou, V Soubassi-Griva

Neonatal Intensive Care Unit, Ippokration Hospital of Thessaloniki, Greece.

A Andreou

Department of Radiology, Ippokration Hospital of Thessaloniki, Greece.

M Pandouraki

Correspondence to Dr Drossou-Agakidou, 52 Neapoleos Street, 54454 Thessaloniki, Greece.

Received for publication 13 December 1990.

Revised version accepted for publication 7 March 1991. years old and non-consanguineous. The parents were clinically and radiologically normal and there was no history of malformations in the family (fig 1) or of exposure to any known teratogenic agents during the pregnancies. Prenatal ultrasound indicated polyhydramnios in both pregnancies, but no malformations were detected. Both pairs of twins were delivered by caesarian section at 38 weeks' gestation.

CASES 1 AND 2 (III-1 AND III $\cdot 2)$

The first pair of twins (cases 1 and 2), two boys, had two placentas and the same $\mathrm{ABO}$ and rhesus blood groups, so that we cannot be certain about the zygosity. Both babies had low Apgar scores and required resuscitation. Endotracheal intubation was impossible and they both received oxygen by face mask and ambu bag. Soon after birth they developed severe respiratory distress which improved after the insertion of nasopharyngeal tubes. Both babies had features of the Pierre-Robin anomalad (severe microretrognathia, glossoptosis, and cleft palate). The chest radiograph of case 1 showed a funnel shaped thorax with 11 pairs of ribs (no 12th pair was identified) and discontinuity of the posterior arcs of both fifth ribs (fig 2 ). The chest radiograph of case 2 was similar with discontinuity of the right fifth rib only (fig 3).

Both babies had clinical signs of mild postasphyxial encephalopathy and feeding difficulties, for which they needed gavage feeding. Case 1 was doing well, breathing through the nasopharyngeal tube, until the 50th day of life, when he was found dead in

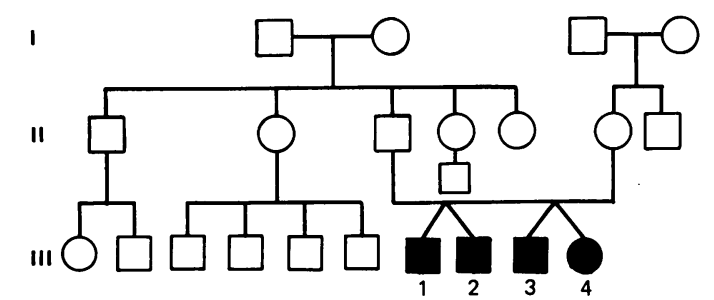

Figure 1 Family pedigree. 


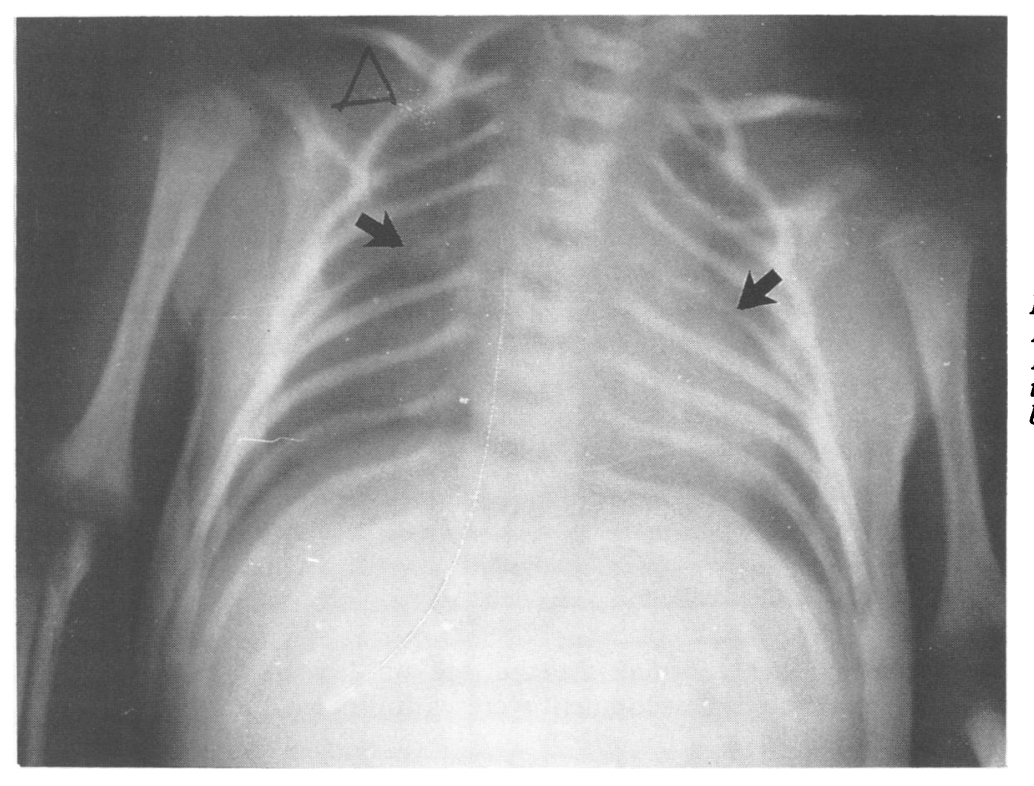

Figure 2 Chest radiograph of case 1 showing funnel shaped thorax with 11 pairs of ribs and discontinuity of the posterior arcs of the fifth ribs bilaterally (arrows).

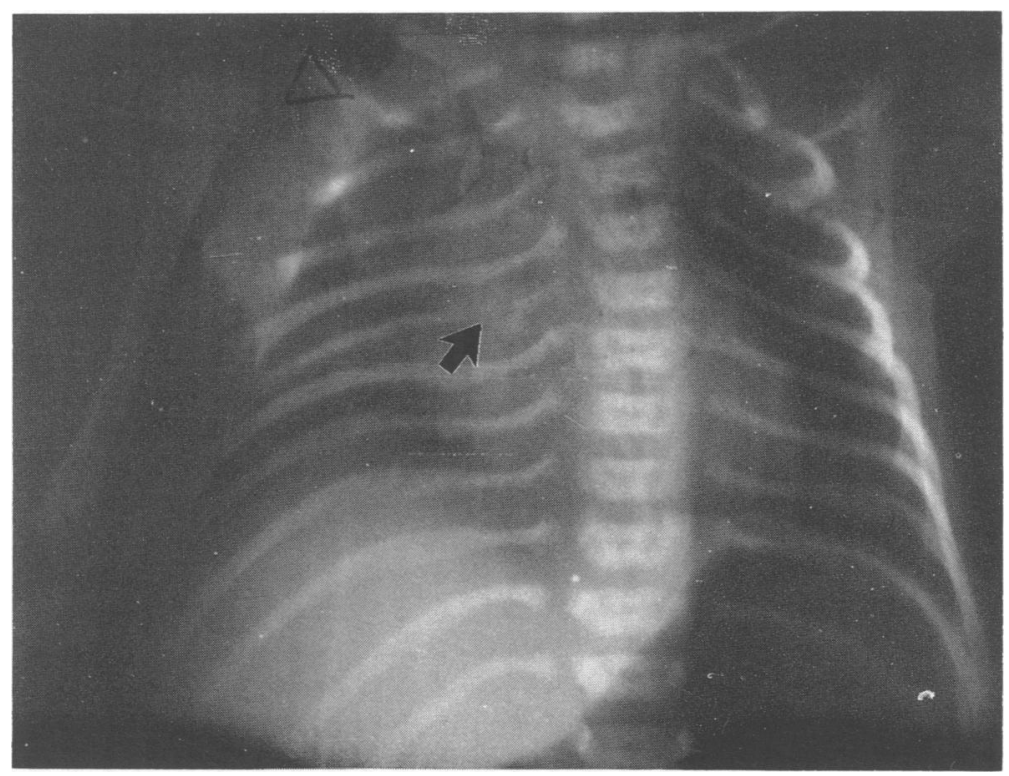

Figure 3 Chest radiograph of case 2 showing 11 pairs of ribs and discontinuity of the fifth right rib (arrow).

his cot. His death was attributed to probable upper airway obstruction. Case 2 developed aspiration pneumonia on the 30th day of life and died five days later in spite of mechanical ventilation. No necropsy was performed.

CASES 3 AND 4 (III 3 AND III 4 )

The second pair of twins, a boy and a girl, were born two years later. Both babies had good Apgar scores, but soon the first one (case 3) developed severe respiratory distress. The respiratory difficulties were not improved by the placement of an oropharyngeal tube. He had a small, bell shaped thorax with abnormal, paradoxical respiratory movements. He also had features of the Pierre-Robin anomalad, redundant skin, and posterior cervical skin fold. Chest radiograph showed severe deformity of the rib cage with only 10 pairs of ribs (no first right, 11th left, or 12th pair of ribs were identified) and multiple 


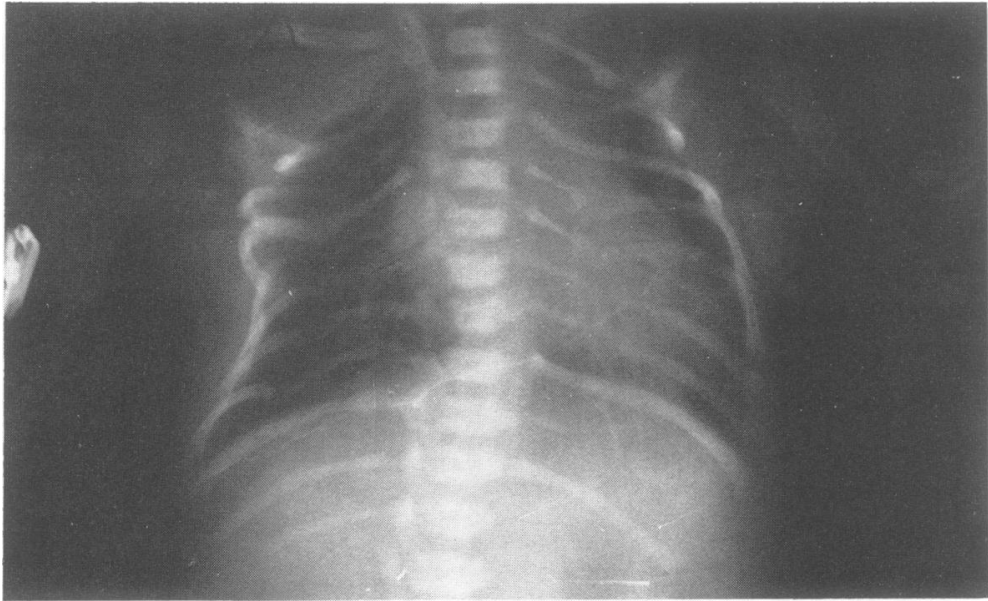

Figure 4 Chest radiograph of case 3 showing severe deformity of the rib cage with 10 pairs of ribs (the first right, 11th left, and both 12th ribs are missing) and multiple discontinuities of the posterior arcs of the right third, fifth, sixth, and eighth ribs and the left fourth, fifth, sixth, seventh, and ninth ribs.

discontinuities of the posterior rib arcs involving the right third, fifth, sixth, and eighth ribs and the left fourth, fifth, sixth, seventh, and ninth ribs (fig 4). The baby died 9 days after birth of intractable respiratory distress. Necropsy showed no other system involvement. Microscopic examination of the ribs was not performed.

Case 4 (III-4) was a girl who had a good Apgar score and no respiratory distress. She had mild features of the Pierre-Robin anomalad (fig 5) and stridor, while her chest radiograph showed 11 pairs of ribs (the 12th pair was missing) and a small discontinuity of the fourth left rib (fig 6). There was no evidence of congenital heart disease and renal ultrasound, brain CT scan, and karyotype were

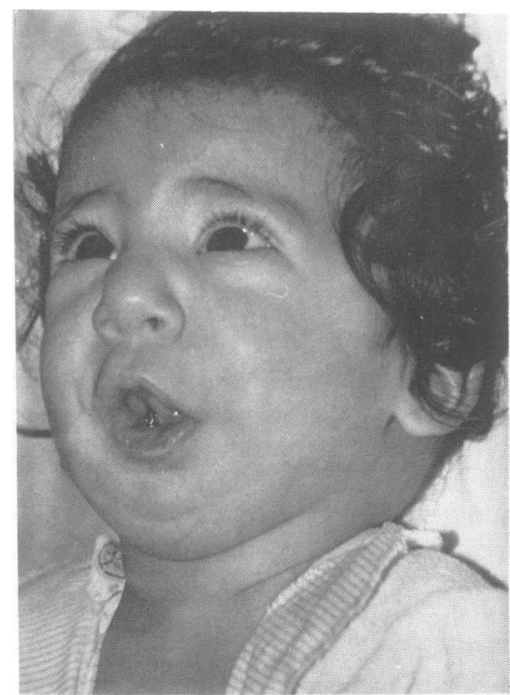

Figure 5 Anterior view of the face of case 4 showing micrognathia and cleft palate. normal. At the age of 2 years her growth and development were within normal limits.

\section{Discussion}

As far as we know these are the first published cases of CCMS in twins. All of our patients had the Pierre-Robin anomalad and the characteristic rib dysplasia of CCMS, but there was great variability in the manifestations of the syndrome. Rib involvement was mild in three patients (cases 1,2 , and 4) and severe in case 3, who died because of severe respiratory distress attributed to severe chest deformity. The Pierre-Robin anomalad was mild in case 4 and severe in cases 1,2 , and 3, contributing to death in cases 1 and 2.

Other features in our cases that have been described before ${ }^{24-6}$ were polyhydramnios, feeding difficulties in three cases, redundant skin and posterior cervical skin fold in one case, and mild stridor in another one. Involvement of the brain is common in CCMS, ${ }^{2}$ but it has been suggested that it is probably caused by perinatal or neonatal hypoxia rather than by a congenital brain defect. ${ }^{57}$ Cerebral involvement in our cases was consistent with acquired cerebral damage, since it was evident only in the first pair of babies, who had suffered perinatal asphyxia. Case 3 had normal findings in the brain on necropsy and case $\mathbf{4}$ had normal brain scan and development at the age of two years.

Most of the reported cases of CCMS have been sporadic, ${ }^{12}$ but familial cases have also been described. ${ }^{3-7}$ Four of the reported familial cases of affected sibs with normal parents are consistent with autosomal recessive inheritance. ${ }^{2367}$ The fully expressed syndrome in one of the parents and their children reported by Leroy et $a l^{5}$ and Merlob et $a^{4}$ indicates autosomal dominant inheritance. ${ }^{45}$ In our cases, the presence of the syndrome in all four of the 


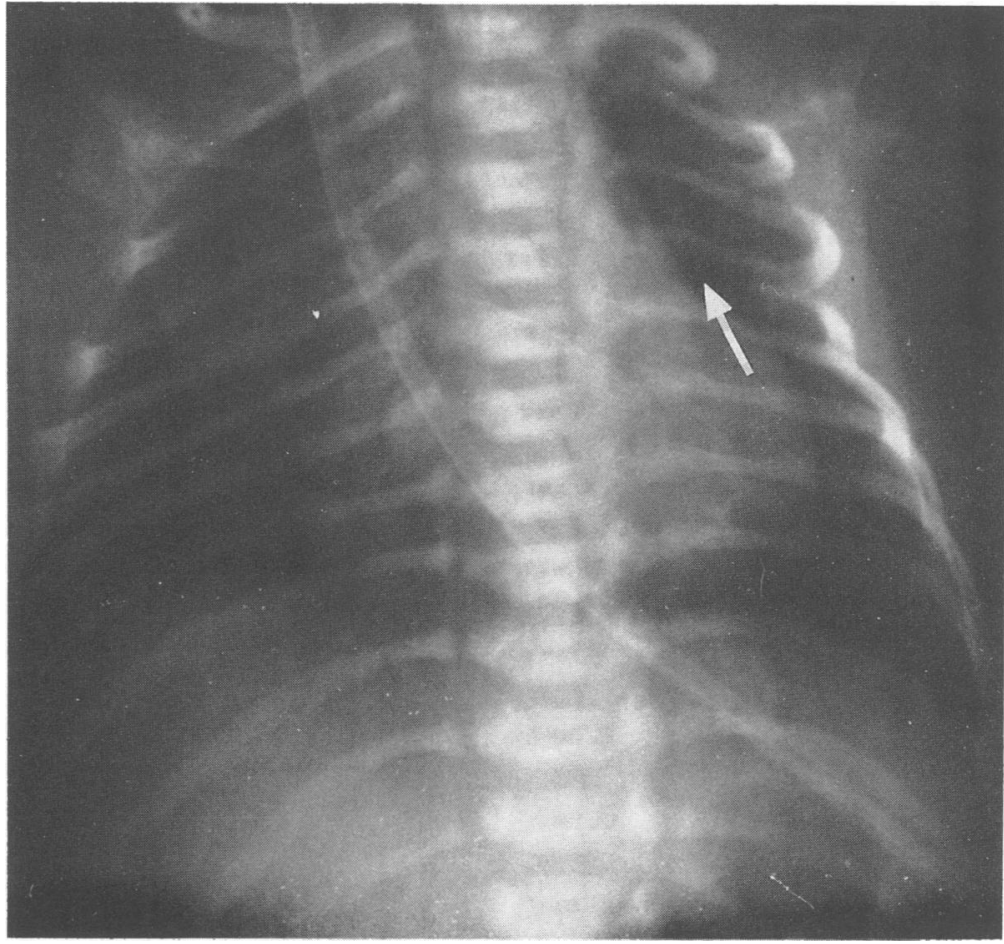

Figure 6 Chest radiograph of case 4 showing 11 pairs of ribs and discontinuity of the fourth left rib (arrow).

two pairs of twins, which means $100 \%$ concordance, ${ }^{8}$ along with the negative family history is consistent with autosomal recessive inheritance and suggests high penetrance of the gene.

1 Smith DW, Theiler K, Schachenmann G. Rib-gap defect with micrognathia, malformed tracheal cartilages, and redundant skin: a new pattern of defective development. $\mathcal{F}$ Pediatr 1966;69:799-803.

2 Silverman FN, Strefling AM, Stevenson DK, Lasarus J. Cerebro-costo-mandibular syndrome. F Pediatr 1980;97:406-16.

3 Hennekam RCM, Huijbers FA, Hustinx PA, VanSprang FJ. The cerebrocostomandibular syndrome: third report of familial occurrence. Clin Genet 1985;28:118-21.
4 Merlob P, Schonfeld A, Grunebaum M, Mor N, Reisner SH. Autosomal dominant cerebro-costo-mandibular syndrome: ultrasonographic and clinical findings. Am $f$ Med Genet 1987;26:195-202.

5 Leroy GJ, Devos EA, Vanden Bulcke LJ, Robbe NS. Cerebrocosto-mandibular syndrome with autosomal dominant inheritance. 7 Pediatr 1981;99:441-3.

6 McNichol B, Egan-Mitchell B, Murray JP, Doyle JE, Kennedy JD, Cromr L. Cerebro-costo-mandibular syndrome. A new familial developmental disorder. Arch Dis Child 1970;45:4214.

7 Trautman MS, Schelley SL, Stevenson DK. Cerebro-costomandibular syndrome: a familial case consistent with autosomal recessive inheritance. $f$ Pediatr 1985;99:990-1.

8 Windam GC, Bjerkedal T. Malformations in twins and their siblings, Norway 1967-1979. Acta Genet Med Gemellol (Roma) 1984;33:87-95. 